\title{
Characterization of class I integrons in clinical strains of Salmonella enterica subsp. enterica serovars Typhimurium and Enteritidis from Norwegian hospitals
}

\author{
Bjørn-Arne Lindstedt, ${ }^{1}$ Even Heir, ${ }^{1}$ Irene Nygård ${ }^{1}$ and Georg Kapperud ${ }^{1,2}$
}

\begin{abstract}
Correspondence
Bjørn-Arne Lindstedt

bjorn-arne.lindstedt@folkehel sa.no
\end{abstract}

Received 7 May 2002

Accepted 9 October 2002

\author{
${ }^{1}$ Norwegian Institute of Public Health, Division of Infectious Diseases Control, N-0403 Oslo, Norway \\ ${ }^{2}$ Department of Pharmacology, Microbiology and Food Hygiene, Norwegian School of Veterinary \\ Sciences, N-0033 Oslo, Norway
}

\begin{abstract}
The characterization of integrons and their promoters in 156 antibiotic-resistant clinical isolates of the important zoonotic pathogens Salmonella enterica subsp. enterica serovars Typhimurium ( $S$. Typhimurium) and Enteritidis (S. Enteritidis) from Norwegian hospitals were performed. Integrons were found in 64 of $66 \mathrm{~S}$. Typhimurium isolates (97\%) and in 20 of $90 \mathrm{~S}$. Enteritidis isolates $(22.2 \%)$ with the following sizes; 650, 1000, 1200, 1500, 1600, 1700, 2000 and $2100 \mathrm{bp}$. The integrons were further sequenced and the $\operatorname{aad} A 1$, aadA2, aad $A 5$, aad $B$, pse-1, catB3, oxa1, dfrA1, $d f r A 12$ and $d f r A 17$ genes, as well as a fragment of the sat 1 gene, were found embedded in cassettes. An internal fragment of the purG gene was additionally found as an artefact PCR amplicon.
\end{abstract}

\section{INTRODUCTION}

Integrons are genetic units that include components of a sitespecific recombination system enabling them to capture and mobilize genes. Integrons contain a gene of the $\lambda$ family of integrases that carries out recombination between two distinct target sites; the attI site and the 59-base element where attI is the target site for cassette integration (Hall \& Collis, 1995; Hall \& Stokes, 1993). The genes captured by this system are packaged as small discrete units with essentially all flanking sequences removed, but with the 59-base element included, and are named 'gene cassettes' (Hall \& Collis, 1995; Hall \& Stokes, 1993). Integrons are believed to be ancient structures (Rowe-Magnus et al., 2001), although the mechanism that created these cassettes is at present unknown. The majority of known gene cassettes are antibiotic resistance genes, but it is likely that any gene could be included in a cassette and several uncharacterized ORFs have been found in cassettes. Integrons are classified by comparison of the amino acid sequence of their integrases, encoded by the int $I$ genes. Four integron types have been identified, but in recent studies additional integron classes have been reported (Rowe-Magnus et al., 2001; Nield et al., 2001). The majority of integrons found in clinical isolates of enterobacteria are of class I. More than 60 different antibiotic resistance genes have been identified within cassette structures either alone or in combination (Mazel \& Davies, 1999).

A promoter region is located in the $5^{\prime}$-conserved segment of the integron and contains two promoters named $\mathrm{P}_{\mathrm{c}}\left(\mathrm{P}_{\mathrm{ant}}\right)$ and
P2. Four different $\mathrm{P}_{\mathrm{c}}$ and two different $\mathrm{P} 2$ promoters have been described in the literature (Stokes \& Hall, 1989; Bunny et al., 1995) and their relative strength as compared to the Escherichia coli tac promoter has been reported (Lévesque et al., 1994; Collis \& Hall, 1995). Two of the $\mathrm{P}_{\mathrm{c}}$ promoters are designated strong (TTGACA- $\mathrm{N}_{17}$-TAAACT) and weak (TGGACA-N $\mathrm{N}_{17}$-TAAGCT), and two are named hybrid promoters, with the strong promoter six times more effective than the E. coli tac promoter. The tac promoter is, however, more efficient than the weak cassette promoter and the two hybrid promoters, TGGACA-N 17 -TAAACT and TTGACA$\mathrm{N}_{17}$-TAAGCT (Lévesque et al., 1994; Collis \& Hall, 1995). The $\mathrm{P} 2$ promoter has an active version TTGTTA- $\mathrm{N}_{17}$ TACAGT, but when the -35 and -10 regions are separated by $14 \mathrm{nt}$, TTGTTA-N $\mathrm{N}_{14}$-TACAGT, there is no $\mathrm{P} 2$ promoter activity.

Integrons are widespread and have been studied in great detail among the Enterobacteriaceae, with a particular focus on the multi-resistant Salmonella enterica subsp. enterica serovar Typhimurium ( $S$. Typhimurium) definitive type (DT) 104, while studies on integrons among Salmonella enterica subsp. enterica serovar Enteritidis ( $S$. Enteritidis) isolates are limited. The incidence of $S$. Enteritidis infections has increased in many countries, and in Europe this serotype is now predominant among human Salmonella isolates. The increase began as early as the mid-1970s and $S$. Enteritidis displaced $S$. Typhimurium as the primary cause of infection by 1990 (Kingsley \& Baumler, 2000; Baumler et al., 2000; 
Guard-Petter, 2001). While other Salmonella serotypes have been shown to experimentally infect eggs, $S$. Enteritidis is the only human pathogen that causes frequent human illness associated with egg contamination (Guard-Petter, 2001). $S$. Enteritidis is therefore an important zoonotic pathogen in which increased resistance to several antibiotics will pose an increased threat.

This study presents the result of characterization of integrons and their promoters in 156 multi-resistant strains of the important zoonotic pathogens $S$. Typhimurium and $S$. Enteritidis from human clinical isolates (hospitalized patients) submitted to the Reference Laboratory for enteropathogenic bacteria at the Norwegian Institute of Public Health from hospitals throughout Norway.

\section{METHODS}

\section{Strain selection}

S. Typhimurium. In all 66 antibiotic-resistant isolates of $S$. Typhimurium from 22 cases of domestically acquired infections and 44 cases of infections acquired abroad, were obtained from the strain collection at the reference laboratory for enteropathogenic bacteria at the Norwegian Institute of Public Health, Oslo, Norway. The strains were isolated from 1996 to 2001. A total of 53 isolates were phage-typed as DT104 and 22 of these had the ACSSuT (see below for definition of abbreviations) resistance profile (Tables 1 and 2).

S. Enteritidis. The strain collection of the National Reference Laboratory had 1114 S. Enteritidis isolates from 2001 of which 27 (2.4\%) were domestically acquired. A total of $54(4 \cdot 8 \%)$ were resistant to two or more antibiotics. In 2000 the strain collection contained $794 \mathrm{~S}$. Enteritidis isolates where $10(1.3 \%)$ were domestically infected and $36(4.5 \%)$ were resistant to two or more antibiotics. All the 90 isolates of $S$. Enteritidis that were resistant to two or more antibiotics were obtained from the strain collection at the reference laboratory. Table 3 lists the integron-positive $S$. Enteritidis isolates.
Antimicrobial resistance. Antimicrobial susceptibility was tested by a tablet diffusion method according to the manufacturer's guidelines (Rosco Diagnostics). The isolates were screened for resistance to ampicillin (A), ciprofloxacin $(\mathrm{Cx})$, tetracycline $(\mathrm{T})$, chloramphenicol (C), nalidixic acid ( $\mathrm{Na}$ ), trimethoprim-sulfamethoxazole (Ts), sulphadiazine $(\mathrm{Su})$, streptomycin (S), gentamicin $(\mathrm{Gn})$ and doxycycline (Dx). Only $S$. Enteritidis strains that were resistant to two or more antibiotics, and $S$. Typhimurium strains resistant to four or more antibiotics were included in the study.

Integron PCR. A number of PCR primers were used for amplification of gene cassettes and genes known to be associated with integrons as well as a gene associated with transposons, including $\operatorname{Tn} 21, \operatorname{Tn} 501$ and Tn1696. These genes include intI1, intI2, intI3, sulI1, qacE $\Delta 1$, merA and tniA. All strains were tested with markers for the integrase gene intIl, the quaternary ammonium compound resistance gene qacE $\Delta 1$ and the sulphonamide resistance gene sulI, as well as for the integrated gene cassettes. Integrons have been located within transposons and all isolates were additionally tested for the presence of the transposase gene tniA that can be associated with integron In2 (Partridge et al., 2001) and the mercury resistance gene merA associated with the transposon $\operatorname{Tn} 21$ as well as others. Primers for amplification of the antibiotic resistance gene floR were included (Table 4). To examine the cassette promoters, an upper primer situated at the $3^{\prime}$ end of the intIl gene coupled with lower primers situated in the aadA1, aadA2, aadB, pse-1, oxa1, dfrA1, dfrA12 and $d f r A 17$ genes were used. See Figs 1 and 2 for the location of the most used primer sets. The primer in the intI1 gene was placed such that the $P_{c}$ and the P2 promoter sequences, as well as the binding site for the integron cassette-A primer, would be included in the amplified PCR product. Furthermore, primers for fully sequencing the middle regions of the longer cassettes were designed from the end sequences generated by the vector-localized T7 and T3 universal primers.

The temperature profile was: $94^{\circ} \mathrm{C}$ denaturation for $5 \mathrm{~min}$ followed by 25 cycles of $94{ }^{\circ} \mathrm{C}$ for $30 \mathrm{~s}, 55^{\circ} \mathrm{C}$ for $30 \mathrm{~s}$ and $72{ }^{\circ} \mathrm{C}$ for $30 \mathrm{~s}$, then a $7 \mathrm{~min}$ extension step at $72{ }^{\circ} \mathrm{C}$ on a Perkin-Elmer GeneAmp PCR system 9700. The primers in the floR, sulII, merA and tniA genes had the same profile but with annealing at $60^{\circ} \mathrm{C}$.

Cloning and sequencing. The PCR amplicons from the cassette primers were cloned directly into the vector PCR-Script Amp using the

Table 1. Characteristics of the S. Typhimurium DT104 isolates

All isolates carry the two integrons containing aadA2 in one and pse-1 in the other, or aadA2 containing integron alone. +, Present; -, absent.

\begin{tabular}{|c|c|c|c|c|c|c|}
\hline merA & intI1 & qacE $\Delta 1$ & floR & sulI & Resistance & No. of isolates \\
\hline- & + & + & + & - & ACST & 2 \\
\hline- & + & + & + & + & ACSSuT & 22 \\
\hline- & + & + & + & + & ACSuT & 2 \\
\hline- & + & + & + & + & ACSSuTGn & 1 \\
\hline- & - & + & + & - & ACST & 1 \\
\hline- & + & + & + & + & ACSSuTCx & 1 \\
\hline- & + & + & + & + & ACSSuTTm & 2 \\
\hline- & + & + & + & + & ACSSuTDx & 14 \\
\hline- & + & + & - & + & ACSSuTDx & 2 \\
\hline- & + & + & + & + & ACSSuTNaDx & 2 \\
\hline- & - & - & - & + & ACSTDx & 1 \\
\hline- & - & + & + & + & ACSSuT & 1 \\
\hline+ & - & + & - & + & ACSSuTDx & 1 \\
\hline- & - & + & + & + & ACSSuTDx & 1 \\
\hline
\end{tabular}


Table 2. Characteristics of the S. Typhimurium non-DT104 isolates

+, Present; -, absent; NR, no reaction; NDA, not domestically acquired, but country of origin uncertain.

\begin{tabular}{|c|c|c|c|c|c|c|c|c|c|}
\hline Isolate & $\begin{array}{c}\text { Year of } \\
\text { isolation }\end{array}$ & $\begin{array}{l}\text { Phage } \\
\text { type }\end{array}$ & intI1 & qacE $\Delta 1$ & merA & Resistance & Location & Genes in cassettes & Plasmid \\
\hline $1864 / 96$ & 1996 & NR & + & + & - & CSTTmGn & India & aadB-catB3 & Yes \\
\hline $1875 / 96$ & 1996 & DT120 & + & + & + & ACSSuT & Spain & oxa1-aadA1 & Yes \\
\hline $1550 / 96$ & 1996 & DT120 & + & + & + & ACSSuT & Spain & oxa1-aadA1 & Yes \\
\hline $1974 / 98$ & 1998 & U302 & + & + & + & ACSSuT & Portugal & aadA2-pse1 & No \\
\hline $900 / 98$ & 1998 & NR & + & + & - & ACSSuTTmGn & Ethiopia & aadB-catB3-oxa1-aadA1 & Yes \\
\hline $3428 / 98$ & 1998 & NR & + & + & + & ACSSuTTmGn & Africa & aadB-catB3-oxa1-aadA1 & Yes \\
\hline $2339 / 99$ & 1999 & DT120 & + & + & + & ACSSuT & Spain & oxa1-aadA1 & Yes \\
\hline $5180 / 00$ & 2000 & DT204 & + & + & + & CSSuTTmDx & Greece & $d f r A 17-a a d A 5$ & Yes \\
\hline
\end{tabular}

Table 3. Characteristics of the $S$. Enteritidis isolates containing integrons

All the integron-positive isolates were positive for intI1, qacE $\Delta 1$ and sulI. All the integrons were localized on plasmids. NDA, Not domestically acquired, but country of origin uncertain.

\begin{tabular}{|c|c|c|c|c|}
\hline Isolate & $\begin{array}{c}\text { Year of } \\
\text { isolation }\end{array}$ & Origin* & Resistance & Genes in cassettes \\
\hline $7228 / 00$ & 2000 & Indonesia & ATNaTs & $d f r A 1-a a d A 1$ \\
\hline $4084 / 00$ & 2000 & Rwanda & ACTs & $d f r A 1-a a d A 1$ \\
\hline $4305 / 00$ & 2000 & India & ATCTs & $d f r A 1-a a d A 1$ \\
\hline $9843 / 00$ & 2000 & $\mathrm{NDA}$ & ATNaTs & $d f r A 1-a a d A 1$ \\
\hline $4027 / 00$ & 2000 & Kenya & ATCTs & $d f r A 1-a a d A 1$ \\
\hline $3635 / 00$ & 2000 & $\mathrm{NDA}$ & TNaTs & $d f r A 1-a a d A 1$ \\
\hline $9417 / 01$ & 2001 & $\mathrm{NDA}$ & TTs & sat1 $\Delta$ \\
\hline $9997 / 01$ & 2001 & Italy & TTs & $d f r A 1-a a d A 1$ \\
\hline $7508 / 01$ & 2001 & Asia & ATs & dfrA12-orf-aadA2 \\
\hline $7224 / 01$ & 2001 & $\mathrm{NDA}$ & TTs & $d f r A 1-a a d A 1$ \\
\hline $1694 / 01$ & 2001 & Spain & TTs & $d f r A 1-a a d A 1$ \\
\hline $5191 / 01$ & 2001 & Thailand & TNaTs & $d f r A 1-a a d A 1$ \\
\hline $1731 / 01$ & 2001 & Iran & ATTs & $d f r A 1-a a d A 1$ \\
\hline $9430 / 01$ & 2001 & Greece & Ts & $d f r A 1-a a d A 1$ \\
\hline $2712 / 01$ & 2001 & Thailand & ATNaTs & $d f r A 1-a a d A 1$ \\
\hline $3121 / 01$ & 2001 & Asia & TNaTs & $d f r A 1-a a d A 1$ \\
\hline $775 / 01$ & 2001 & Spain & TTs & $d f r A 1-a a d A 1$ \\
\hline $3120 / 01$ & 2001 & South America & TNaTs & dfrA12-orf-aadA2 \\
\hline $5693 / 01$ & 2001 & Spain & TTs & $d f r A 1-a a d A 1$ \\
\hline 4947/01 & 2001 & France & TTs & $d f r A 1-a a d A 1$ \\
\hline
\end{tabular}

PCR-Script Amp Electroporation-competent Cell Cloning Kit (Stratagene) after purification with the QIAquick PCR purification kit (Qiagen). In some cases bands were cut out from the agarose gel and DNA was extracted with the QIAquick gel extraction kit (Qiagen) before cloning. The ligated vector + amplicon construct was transformed into Epicurian Coli XL-1 Blue MRF' electroporation competent cells
(Stratagene) with a Gene Pulser II (Pulse Controller Plus; Bio-Rad) set at $400 \Omega$ resistance, $2.5 \mathrm{kV}$ voltage and $25 \mu \mathrm{F}$ capacitance using a $0.2 \mathrm{~cm}$ cuvette. The transformed cells were incubated for $1 \mathrm{~h}$ at $37^{\circ} \mathrm{C}$ in SOC medium before plating on IPTG/X-Gal plates with $50 \mu \mathrm{g}$ ampicillin $\mathrm{ml}^{-1}$ for blue-white screening of positive inserts. The plates were incubated overnight at $37^{\circ} \mathrm{C}$ and cooled $1 \mathrm{~h}$ at $4{ }^{\circ} \mathrm{C}$ to intensify the blue- 
Table 4. PCR primer pairs

\begin{tabular}{|c|c|c|}
\hline Primer & Sequence $\left(5^{\prime}-3^{\prime}\right)$ & Reference \\
\hline cassette-A & GGCATCCAAGCAGCAAG & Lévesque et al. (1995) \\
\hline cassette-B & AAGCAGACTTGACCTGA & Lévesque et al. (1995) \\
\hline intI1-F & GCCTTGCTGTTCTTCTACGG & Lévesque et al. (1995) \\
\hline intI1-R & GATGCCTGCTTGTTCTACGG & Lévesque et al. (1995) \\
\hline intI2-F & CACGGATATGCGACAAAAAGGT & Mazel et al. (2000) \\
\hline intI2-R & GTAGCAAACGAGTGACGAAATG & Mazel et al. (2000) \\
\hline intI3-F & GCCTCCGGCAGCGACTTTCAG & Mazel et al. (2000) \\
\hline intI3-R & ACGGATCTGCCAAACCTGACT & Mazel et al. (2000) \\
\hline sulI1-F & CTTCGATGAGAGCCGGCGGC & Sandvang et al. (1997) \\
\hline sulI1-R & GCAAGGCGGAAACCCGCGCC & Sandvang et al. (1997) \\
\hline qacE $\Delta 1-F$ & ATCGCAATAGTTGGCGAAGT & Sandvang et al. (1997) \\
\hline qacE $\Delta 1-R$ & CAAGCTTTTGCCCATGAAGC & Sandvang et al. (1997) \\
\hline merA-F & ATCGGCGGCACCTGCGTCAATGTC & This study \\
\hline merA-R & CGGCCAGGCGCTTAGGAATCGTCT & This study \\
\hline floR-U & ACCCGCCCTCTGGATCAAGTCAAG & This study \\
\hline floR-L & CAAATCACGGGCCACGCTGTATC & This study \\
\hline $\operatorname{tniA}-\mathrm{U}$ & GCTCGCGCCGGGCAATCCAC & This study \\
\hline tniA-L & CCCACCAGGCCAGCCGCACTACG & This study \\
\hline IPUR-U & CGGTGTGGCGGGCTTCGTGATG & This study \\
\hline IPUR-L & CCTCGCCGCTGTCGCTGGTGATT & This study \\
\hline pse-1-R & GGGGCTTGATGCTCACTCCA & Huovinen \& Jacoby (1991) \\
\hline pse-1-L & AATGGCAATCAGCGCTTCCC & Huovinen \& Jacoby (1991) \\
\hline aadA2-L & TGTTGGTTACTGTGGCCGTA & Bito \& Susani (1994) \\
\hline aadA2-R & GCTGCGAGTTCCATAGCTTC & Bito \& Susani (1994) \\
\hline
\end{tabular}

$5^{\prime}$ conserved segment

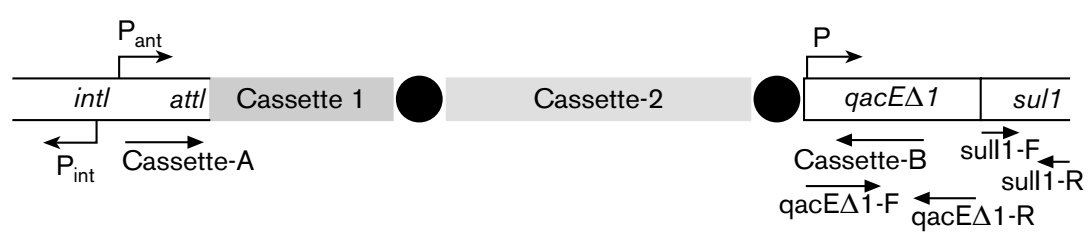

$500 \mathrm{bp}$ $3^{\prime}$ conserved segment

Fig. 1. Location of the PCR primers used to amplify the integron variable regions containing the inserted gene cassettes (cassette- $A$ and cassette-B), the primer set to amplify the disinfectant resistance gene qacE $\Delta 1$ and the primer set for the sull1 gene. Black circles denote the location of the 59-base element, attl is the cassette integration site and $\mathrm{P}$ denotes promoters.

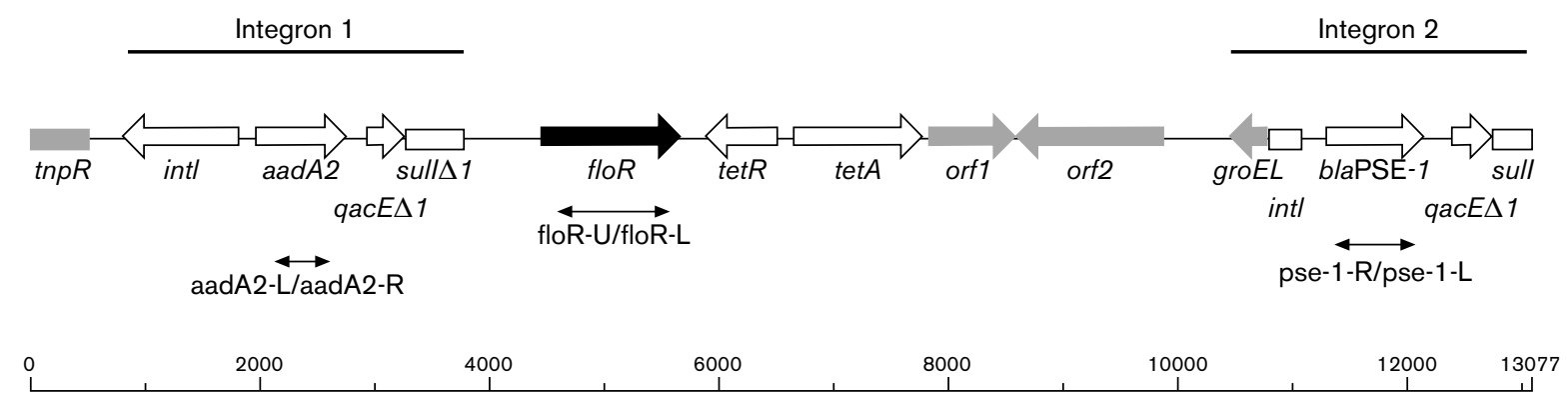

Fig. 2. Location of the PCR primers used to amplify genes associated with the S. Typhimurium DT104 integron region. The genes are aadA2, floR and pse- 1 . 
coloured colonies. White colonies were picked and incubated overnight in LB medium with $50 \mu \mathrm{g}$ ampicillin $\mathrm{ml}^{-1}$ added. Plasmids were then isolated with the Wizard Plus SV Minipreps DNA Purification System (Promega). All the isolated plasmids were digested for $1 \mathrm{~h}$ at $37^{\circ} \mathrm{C}$ with the restriction endonucleases PstI and NotI (New England Biolabs) and separated on a $1 \%$ GTG agarose gel to confirm insert sizes. Plasmids containing inserts were subjected to cycle sequencing with the BigDye Terminator kit (ABI biosystems) from both ends with the T7 and T3 universal sequencing primers. The sequence reaction was separated by capillary electrophoresis on an ABI-310 Genetic Analyser (ABI biosystems) with POP- 6 polymer. Some of the amplicons from the cassette primers were in addition subjected to direct sequencing after purification with the QIAquick PCR Purification kit (Qiagen) using the BigDye Terminator kit (ABI biosystems) with $3 \cdot 2$ pmol of either the cassette-A or cassette-B primer (Table 4). All sequencing reactions were purified with the DyeEx Spin Kit (Qiagen) to remove unincorporated dye terminators prior to electrophoresis. The promoter regions for all the found cassette amplicons were subjected to direct sequencing of both strands with $3 \cdot 2 \mathrm{pmol}$ of the appropriate primer sets.

Sequence analysis. The sequence raw data were exported from the ABI-310 into the Lasergene software package (DNASTAR). All sequence comparisons and alignments were done using this software which additionally performed on-line searches for related sequences via the National Center for Biotechnology Information (NCBI) BLAST server.

Plasmid isolation and hybridization. Plasmids were isolated from all strains as detailed by Kapperud et al. (1989) and separated by $1 \%$ agarose gel electrophoresis. The gels were photographed under UV light after ethidium bromide staining. Plasmid DNA was transferred to Hybond-N+ membranes (Amersham-Pharmacia Biotech) by vacuum blotting and hybridized at $42{ }^{\circ} \mathrm{C}$ using synthetic oligonucleotide probes directed at the intI1, sulI and qacE $\Delta 1$ genes with the ECL 3 '-oligolabelling and detection system (Amersham-Pharmacia Biotech).

\section{RESULTS AND DISCUSSION}

\section{Integron markers}

Sixty-six antibiotic-resistant $S$. Typhimurium and $90 \mathrm{~S}$. Enteritidis isolates, all of human origin, were tested for the presence of known markers for integrons by PCR amplification. The integrase intI1 gene was detected in $91 \%(60 / 66)$ of the $S$. Typhimurium isolates; only strains 10403, 700/98, $5031 / 00,6347 / 00,4805 / 00$ and 5790/00 failed to give an amplicon after repeated attempts with the intI1 primer set. The isolates were tested with primers for two additional known integron integrases, intI2 and intI3, which both failed to amplify a fragment. In the $S$. Typhimurium isolates 5031/ 00 and 6347/00 no amplicons from the gene cassette primers were detected. In isolates 10403, 700/98, 4805/00 and 5790/ 00 gene cassettes were found with the cassette primers, indicating a possible deletion in the intI1 gene upstream from the $5^{\prime}$ conserved segment cassette primer-binding site, or the presence of a different class of integrase. The most common amplicons were 1000 and $1200 \mathrm{bp}$ that predominantly occurred in combination and were detected in 51 of the $66(77 \cdot 3 \%)$ S. Typhimurium isolates. The $1000 \mathrm{bp}$ amplicon occurred alone in two $S$. Typhimurium isolates $(3 \%)$ and the remaining $S$. Typhimurium amplicons had sizes of 200,1500, 1600, 1700, 2000 or $2100 \mathrm{bp}$. A notable feature is that the vast majority of $S$. Typhimurium isolates carried two gene cassettes; only $10 \mathrm{~S}$. Typhimurium isolates $(15 \cdot 2 \%)$ displayed single bands from the cassette primers. The disinfectant resistance gene qacE $\Delta 1$ was absent in one $S$. Typhimurium isolate $(1.5 \%)$ and the sulphonamide resistance gene sulI was absent in seven $S$. Typhimurium isolates (10.6\%). Only one $S$. Typhimurium (1.5\%) isolate (2755/ 97) was positive for the tniA gene which has been associated with the In0, In2, In5 and Tn402(In16) integrons (Radstrom et al., 1994; Brown et al., 1996; Hall et al., 1994).

All the integron-positive $S$. Enteritidis isolates (Table 3) contained the intIl, qacE $\Delta 1$ and sulI genes. The merA marker was found in all the $S$. Enteritidis isolates with the dfrA1aadA1 integron, but none of the $S$. Enteritidis isolates were positive for the tniA marker.

\section{S. Typhimurium gene cassettes}

All the $1000 \mathrm{bp}$ amplicons were shown to contain the aadA2 gene that confers resistance to streptomycin and spectinomycin with a perfect match ( $100 \%$ similarity) to the aadA2 gene from S. Typhimurium DT104 (accession no. AF071555; Briggs \& Fratamico, 1999). The $1200 \mathrm{bp}$ amplicons contained the pse- $1 \beta$-lactamase gene with a perfect match to the pse-1 gene from $S$. Typhimurium DT104 (accession no. AF071555; Briggs \& Fratamico, 1999). The aadA2 gene cassette was seen alone in two strains (4380/00 and 7192/ 00 ) which also failed to amplify products with $p s e-1$-specific primers. The pse-1 cassette thus appears to be lost in these strains. No isolates carried the pse-1 gene cassette alone without an accompanying aadA2 cassette. The $1500 \mathrm{bp}$ amplicon was found to carry two gene cassettes in tandem, consisting of the $a a d B$ and the catB3 genes with a perfect match to the integron In-t1 (accession no. AJ009818; Tosini et al., 1998). The 2000 bp amplicon carried two known genes, $d f r A 12$ and aadA2, as well as an ORF and was present in a DT104a isolate. This integron is discussed below in the $S$. Enteritidis section. The $2100 \mathrm{bp}$ amplicon carried two tandemly inserted gene cassettes consisting of the oxal and the aadA1 genes with a perfect match to the In-t2 integron (accession no. AJ009819; Tosini et al., 1998). Tosini et al. (1998) found a third integron (In-t3) of 3200 bp that was localized to an IncL/M plasmid. This $3200 \mathrm{bp}$ integron was absent in our isolates, which appear to contain only the IncFI plasmid. The $1700 \mathrm{bp}$ amplicon revealed two gene cassettes consisting of the $d f r A 17$ and the aadA5 genes. The $1600 \mathrm{bp}$ amplicon contained gene cassettes with the $d f r A 1$ and aadA1 genes and was found in one isolate (7542/00) from Belgium. This $d$ frA1-aadA1-containing integron was confirmed to be plasmid-located.

\section{S. Typhimurium integron-combinations and structure}

The most common feature in $S$. Typhimurium was the presence of two integrons, one containing aadA2 and the other the pse-1 gene. This combination has been observed several times and is characteristic for the genetically homogeneous $S$. Typhimurium DT104 strain which has emerged 
to become the most common type of $S$. Typhimurium isolate in several countries and is predominantly associated with resistance to multiple antibiotics. We failed to get any integron variable region amplicons for only two isolates (5031/00 and 6347/00) using the cassette primers, but isolate $6347 / 00$ was positive for both aadA2 and pse- 1 when primers directed at these genes were used, and isolate 5031/00 was positive for aadA2. It is likely that these genes are located as cassettes, but deletions in the $5^{\prime}$ integron structures that affect the upper cassette primer possibly prevent PCR amplification. These two strains also carried the integronassociated $q a c E \Delta 1$ gene and one strain $(6347 / 00)$ also carried the sull gene, which may further indicate the presence of a $5^{\prime}$ deleted integron structure. Another possible, but less likely explanation would be that the aadA2 and pse- 1 genes in these isolates are not localized within integrons. Isolate 5031/00 (DT104) is additionally negative for the sulI and the floR genes, which again suggest extensive deletions in the integron region of this isolate. Both domestically acquired isolates and isolates from patients that have been abroad show the same dominance of the DT104 integron combination as would be expected from the known frequency of this phage type worldwide. The $1600 \mathrm{bp}$ amplicon from isolate $7542 / 00$ with the $d f r A 1$-aadA1 gene combination, conferring resistance to trimethoprim and streptomycin, has recently been seen in Irish $S$. Typhimurium animal and food isolates (Daly \& Fanning, 2000) as well as in Norwegian animal isolates (Sunde \& Sørum, 1999). Two isolates (3428/98 and 900/98, Table 2) from infants that had returned from travelling in Africa showed two recently published integron structures, In-t1 and In-t2 (Tosini et al., 1998) with sizes of 1500 and $2100 \mathrm{bp}$, respectively. These two integrons have previously been identified in $S$. Typhimurium isolates from Albanian infants with acute gastroenteritis (Tosini et al., 1998). The $1500 \mathrm{bp}$ In-t 1 amplicon contained the $\mathrm{aad} B$ gene that has been shown to confer resistance to kanamycin and low levels of gentamicin (Shaw et al., 1993), and the catB3 gene that encodes the enzyme chloramphenicol acetyltransferase (CatB3) (Bunny et al., 1995). The 2100 bp In-t2 amplicon contained the oxa1 gene that encodes a $\beta$-lactamase and the aadA 1 gene that encodes the aminoglycoside adenyltransferase $\mathrm{AAD}\left(3^{\prime \prime}\right)$-Ia enzyme which confers streptomycin resistance. These two integrons were both located on an IncFI plasmid in the Albanian isolates (Tosini et al., 1998; Carattoli et al., 2001), and also in our two isolates the integrons were confirmed by plasmid isolation and hybridization to be located on plasmids. This is the second report confirmed by sequencing of $S$. Typhimurium isolates that carry a plasmid with both In-t1 and In-t 2 where all isolates in both studies originated from infants with gastroenteritis. One isolate (1864/96) carried only a plasmid-borne In-tl integron. This was a 28 -year-old patient infected in India. The second IncL/ $\mathrm{M}$ plasmid was absent in our isolates with the In-t 1 and In-t2 integrons. This can possibly be ascribed to the higher conjugation frequency of the IncFI plasmid as compared to the IncL/M plasmid, giving it a higher potential to transfer between isolates. Isolate $3428 / 98$ is additionally positive for the mercury resistance gene merA that is associated with transposon Tn21 and some other known transposons, while isolate $900 / 98$ is not. The In-t2 integron of $3428 / 98$ is thus likely to be part of a larger transposon structure. The $200 \mathrm{bp}$ amplicon was not consistently detected and was both present and absent in parallels of the same isolate, and after gel electrophoresis this $200 \mathrm{bp}$ amplicon was always a fainter band than the amplicons of $1000 \mathrm{bp}$ or longer. The $200 \mathrm{bp}$ amplicon contained an ORF that was an internal segment of the purG gene encoding phosphoribosylformylglycinamide synthetase and has been described as a small integron in $S$. Typhimurium isolates from the Republic of Ireland amplified with the cassette primers (Daly et al., 2000). We found that this $200 \mathrm{bp}$ amplicon was a PCR artefact on closer examination of the sequence and after negative test PCRs with a primer set with one primer in the intI1 gene and one in the pur $G$ fragment (IPUR-U/IPUR-L) (Table 4). The absence of the disinfectant gene qacE $\Delta 1$ in one strain and the sulphonamide resistance gene in seven, indicates a possible increase in gene loss at the integron $3^{\prime}$-conserved segment region as noted by Hall et al. (1994). Isolate $8709 / 00$ is a DT104 strain with an ACSSuT resistance profile, but the Pse1 protein in this isolate is truncated as a consequence of a frameshift caused by a T insertion in the coding sequence just downstream for the ATG initiation codon. This truncated protein is believed to be non-functional. The $8709 / 00$ isolate is still resistant to ampicillin which can only be explained by the presence of another functional $\beta$-lactamase, either chromosomal or plasmid-borne. The integron in strain $5180 / 00$ was shown to contain the $d f r A 17$ and aadA5 genes. The $d f r A 17$ gene confers resistance to trimethoprim and the recently described aadA5 gene (Sandvang, 1999) confers resistance to spectinomycin, but not to streptomycin (White et al., 2000). A sequence homology search in GenBank revealed three homologous integron sequences where two were from $E$. coli isolates and one was an unpublished sequence from a Klebsiella pneumoniae isolate. The first $E$. coli dfrA17-aadA5 integron was from Australia and most likely had a chromosomal location (accession no. AF169041), the strain having been isolated from the urinary tract of a hospitalized patient (White et al., 2000). The other E. coli dfrA17-aadA5 integron was from a clinical urine specimen isolated in Taiwan; this integron (accession no. AF170088) was located on a $119 \mathrm{~kb}$ transferable plasmid (Chang et al., 2000a). The third homologue to the 5180/00 $d f r A 17$-aadA5 integron was an unpublished sequence from a K. pneumoniae isolate (accession no. AF220757). The 5180/ $00 \mathrm{~S}$. Typhimurium strain was isolated from a patient infected in the Mediterranean region and carries the merA gene, which suggests that this integron could be transposonassociated. The plasmid preparation from strain 5180/00 in our study was negative for the integron-associated intI1, qacE $\Delta 1$ and sulI genes, making the integron most likely chromosomal in our isolate. This appears to be the first finding of this integron structure in $S$. Typhimurium, and the possible association with a merA-containing transposon will facilitate its further movement between strains.

When we only considered the non-DT104 isolates we 
detected the $a a d B$-catB3 integron in three isolates, the oxa1aadA1 integron in five isolates, the aadA2 integron in two isolates together with the pse-1 integron, the $d f r A 17$-aadA5 integron in one isolate and the dfrA12-orf-aadA2 and $d f r A 1$ aadA1 integrons also in one isolate each. The pse-1 sequence in the $1200 \mathrm{bp}$ cassette of strain $8709 / 00$ displayed an insertion of an extra T nucleotide at nt 466 that would cause a frameshift and thus early termination at nt 510 in the 8709 / 00 pse- 1 gene as compared to the pse-1 sequence from GenBank (accession no. AF313472).

\section{S. Enteritidis integrons}

In this report we additionally describe the presence of class I integrons among isolates of the important zoonotic pathogen $S$. Enteritidis from hospitals throughout Norway, isolated from 2000 to 2001, which makes them suited for describing the present situation concerning the frequency of integron-containing isolates of $S$. Enteritidis in Norway. The level of $S$. Enteritidis infections acquired domestically is very low in Norway. The majority of patients with illness caused by $S$. Enteritidis are infected abroad. Twenty of the investigated resistant isolates of $S$. Enteritidis contained integrons $(22.2 \%)$; in $200016.7 \%(6 / 36)$ of the isolates contained integrons while in 2001 this number had risen to $25.9 \%$ (14/ $54)$.

Gene cassette amplicons of 650, 1600 and $2000 \mathrm{bp}$ were found. The $650 \mathrm{bp}$ amplicon was shown to contain a fragment of the sat1 gene, which in its full version gives resistance to streptothricin (Tschape et al., 1984; Heim et al., 1989). The $1600 \mathrm{bp}$ amplicons were found to contain two integron gene cassettes, $d f r A 1$ and aadA1. The $2000 \mathrm{bp}$ amplicons were found to carry an array of three cassettes, $d f r A 12$-orf-aadA2. The $d f r A 12$ gene encodes a variant of the dihydrofolate reductase gene and the aadA2 gene confers resistance to streptomycin. The $d f r A 12$ gene product confers a 10-times higher level of resistance to trimethoprim than the most common trimethoprim resistance gene $d f r a A 1$ (Heikkila et al., 1993). The dfrA12-orf-aadA2 gene cluster has previously been seen in several strains, including an E. coli O157 isolate (GenBank accession no. AF335108, unpublished), a Serratia marcescens isolate (GenBank accession no. AF284063, unpublished), a Spanish $S$. Typhimurium phage type U302 isolate (Guerra et al., 2001; Echeita et al., 2001), an E. coli Shiga-toxin producing O111: $\mathrm{H} 8$ isolate from the USA (Zhao et al., 2001), E. coli isolates from Taiwan (Chang et al., 2000b), E. coli and Shigella isolates from Finland (Heikkila et al., 1993), an epidemic Vibrio cholerae O1 strain from Guinea-Bissau (Dalsgaard et al., 2000) and in an S. Typhimurium DT104a isolate originating from Australia (this report).

The results showed that integrons containing resistance to trimethoprim and streptomycin were frequently associated with multi-resistant $S$. Enteritidis isolates. Since the level of $S$. Enteritidis isolates containing resistance to two or more antibiotics is only about $4 \%$ of the total isolates, the number of these mobile genetic elements brought into Norwegian hospitals by $S$. Enteritidis is still modest. Our results, however, indicate a possible increase in the integron-containing isolates in Norwegian hospitals from 2000 to 2001. The frequency of integron carriers among the studied isolates rose from $17 \%$ in 2000 to $25.9 \%$ in 2001. In a study of antimicrobial resistance in $S$. Enteritidis isolated between 1990 and 1998 in Southern Italy it was reported that 44 of 1889 strains $(2 \cdot 3 \%)$ were resistant to at least one antibiotic (Nastasi et al., 2000). Of these 44 isolates, six were shown to carry integrons. This gives a frequency of integron carriers among resistant isolates of $13.6 \%(6 / 44)$ in the strains isolated between 1990 and 1998 (Nastasi et al., 2000). Our data indicate that this number might be increasing, and our frequency of about $22 \%$ of resistant isolates carrying integrons is most likely a slight underestimate of the total frequency, since we have only looked at isolates containing resistance to two or more antibiotics. In a screening of all sulfamethoxazole-resistant $S$. Enteritidis isolates received by the Scottish Salmonella Reference Laboratory in 1997, integrons were detected in $19 \%$ of the isolates (11/58) (Brown et al., 2000), which is a frequency comparable to our data for 2000 of about $17 \%$. It will be interesting to see whether our increase in integron-carrying $S$. Enteritidis isolates from 2000 to 2001 will continue or if this was a fluctuation. Isolates from 2002 are being screened to address this in our laboratory. The only integron found in the 2000 isolates was the $d f r A 1$-aadA1 integron, while in 2001 we also detected the aadA2 and $d f r A 12$ genes in addition to a fragment of the sat1 gene in $S$. Enteritidis.

All $S$. Enteritidis integrons in this study were localized on plasmids, indicating that they contain the potential to spread further after introduction to a hospital environment.

\section{Integron cassette promoters}

The promoter regions in front of all the gene cassettes were PCR-amplified and sequenced. The promoters were homogeneous with all isolates showing both $\mathrm{P}_{\mathrm{c}^{-}}$(also called $\mathrm{P}_{\mathrm{ant}}$ ) and P2-related sequences. Only one variation of the $\mathrm{P}_{\mathrm{c}}$ promoter was found among all the integrons sequenced. The majority of samples had the $\mathrm{P}_{c}$ promoter sequence TGGACA-N $\mathrm{N}_{17}$-TAAGCT, irrespective of the cassette following this promoter (Table 5). Only the 5180/00 S. Typhimurium isolate had a variant $\mathrm{P}_{\mathrm{c}}$ promoter with the sequence TGGACA-N $\mathrm{N}_{17}$-TAAACT in front of the $d f \mathrm{rA} 17$-aadA5 gene cassettes (Table 5). In the P2-related sequences we found that all but seven isolates had the sequence TTGTTA- $\mathrm{N}_{14}$ TACAGT, which is not a functional $\mathrm{P} 2$ promoter. The $S$. Typhimurium isolates 1875/96, 1550/96, 1203/97, 2339/99, $1176 / 96$ and $900 / 98$ had all active P2 promoters where the distances between the -35 and the -10 regions of the P2 promoters were $17 \mathrm{nt}$ (Table 5). All these isolates displayed active $\mathrm{P} 2$ promoters in front of an oxa1-aadA1 gene cassette (Table 5). We thus found that the majority of $\mathrm{P} 2$ promoter signals in the In-t2 integrons (oxa1-aadA1) have a spacing between the -10 and -35 regions that is optimal for promoter function. These In- $\mathrm{t} 2$ isolates are thus believed to carry two active promoters $\left(\mathrm{P}_{\mathrm{c}}\right.$ and $\left.\mathrm{P} 2\right)$. This is in accordance 
Table 5. Integron cassette promoters

Nucleotides in italic are only P2-related sequences and are not part of an active promoter.

\begin{tabular}{|c|c|c|c|c|c|c|c|}
\hline \multirow[t]{3}{*}{ Isolate } & \multicolumn{6}{|c|}{ Promoter sequences } & \multirow[t]{3}{*}{ Type } \\
\hline & \multicolumn{3}{|c|}{$\mathbf{P}_{\mathrm{C}}$} & \multicolumn{3}{|c|}{ P2 } & \\
\hline & -35 & $\mathbf{N}$ & -10 & -35 & $\mathbf{N}$ & -10 & \\
\hline Majority & TGGACA & 17 & TAAGCT & TTGTTA & $14^{*}$ & TACAGT & Weak \\
\hline $5180 / 00$ & TGGACA & 17 & TAAACT & TTGTTA & $14^{*}$ & TACAGT & Hybrid \\
\hline $3428 / 98$ & TGGACA & 17 & TAAGCT & TTGTTA & $14^{*}$ & TACAGT & Weak \\
\hline $900 / 98$ & TGGACA & 17 & TAAGCT & TTGTTA & 17 & TACACA & Weak + mutated P2 \\
\hline $1875 / 96$ & TGGACA & 17 & TAAGCT & TTGTTA & 17 & TACAGT & Weak+P2 \\
\hline $1550 / 96$ & TGGACA & 17 & TAAGCT & TTGTTA & 17 & TACAGT & Weak+P2 \\
\hline $1203 / 97$ & TGGACA & 17 & TAAGCT & TTGTTA & 17 & TACAGT & Weak+P2 \\
\hline $2339 / 99$ & TGGACA & 17 & TAAGCT & TTGTTA & 17 & TACAGT & Weak+P2 \\
\hline $1176 / 96$ & TGGACA & 17 & TAAGCT & TTGTTA & 17 & TACAGT & Weak+P2 \\
\hline $4805 / 00$ & TGGACA & 17 & TAAGCT & TTGTTA & $14^{*}$ & $T-C A G T$ & Weak \\
\hline
\end{tabular}

*A spacing of 14 nucleotides between the -35 and -10 signal is outside the range of functional cassette promoters and these sequences do not constitute active promoters.

with the study by Tosini et al. (1998) (accession no. AJ009819) that also shows a spacing of $17 \mathrm{nt}$ between the -10 and -35 regions in the In- $\mathrm{t} 2 \mathrm{P} 2$ promoter from plasmid IncF/97, and a spacing of $14 \mathrm{nt}$ between the $\mathrm{P} 2$ like -10 and -35 sequences in In-t1 (aadB-catB3) (accession no. AJ009818), similar to our results. Variations were noted, however: for example, the In-t2 integrons of isolate 3428/98 which had a spacing of $14 \mathrm{nt}$ between the P2-like -10 and -35 signals, and isolate 900/98 which had a mutation in the P2 promoter sequence, TTGTTA- $\mathrm{N}_{17}$-TACACA (the last $2 \mathrm{nt}$ changed from GT) (Table 5). According to Carattoli et al. (2001) the In-t2 integron is likely to have been maintained unaltered in IncFI plasmids for nearly 30 years, while the plasmids with both In-t1 and In-t2 (IncFI/97) are considered to be new variants (Carattoli et al., 2001). It thus appears as though the IncFI/97-associated In-t2 integron exists in two variant forms, one where both promoters are active and one where only $\mathrm{P}_{c}$ is active. The In-t2 integron with only a functional $P_{c}$ promoter (strain 3428/98) is believed to exhibit weaker expression of the gene cassettes. The strength of the mutated P2 promoter in 900/98 is unknown and will be investigated further.

All of the $S$. Enteritidis promoters consisted of only the TGGACA- $\mathrm{N}_{17}$-TAAGCT $\mathrm{P}_{\mathrm{c}}$ promoter, which is identical to the most common $S$. Typhimurium $\mathrm{P}_{\mathrm{c}}$ promoters, and did not have a functional $\mathrm{P} 2$ promoter.

\section{ACKNOWLEDGEMENTS}

Dr Truls Leergaard is gratefully acknowledged for providing data regarding antibiotic resistance of the $S$. Typhimurium isolates, and the staff of the National Salmonella Reference Laboratory is gratefully acknowledged for technical assistance and for providing data regarding antibiotic resistance of the $S$. Enteritidis isolates.

\section{REFERENCES}

Baumler, A. J., Hargis, B. M. \& Tsolis, R. M. (2000). Tracing the origins of Salmonella outbreaks. Science 287, 50-52.

Bito, A. \& Susani, M. (1994). Revised analysis of aadA2 gene of plasmid pSa. Antimicrob Agents Chemother 38, 1172-1175.

Briggs, C. E. \& Fratamico, P. M. (1999). Molecular characterization of an antibiotic resistance gene cluster of Salmonella typhimurium DT104. Antimicrob Agents Chemother 43, 846-849.

Brown, A. W., Rankin, S. C. \& Platt, D. J. (2000). Detection and characterisation of integrons in Salmonella enterica serotype enteritidis. FEMS Microbiol Lett 191, 145-149.

Brown, H. J., Stokes, H. W. \& Hall, R. M. (1996). The integrons In0, In2, and In 5 are defective transposon derivatives. J Bacteriol 178, 4429-4437.

Bunny, K. L., Hall, R. M. \& Stokes, H. W. (1995). New mobile gene cassettes containing an aminoglycoside resistance gene, aacA7, and a chloramphenicol resistance gene, catB3, in an integron in pBWH301. Antimicrob Agents Chemother 39, 686-693.

Carattoli, A., Villa, L., Pezzella, C., Bordi, E. \& Visca, P. (2001). Expanding drug resistance through integron acquisition by IncFI plasmids of Salmonella enterica Typhimurium. Emerg Infect Dis 7, $444-447$.

Chang, C. Y., Chang, L. L., Chang, Y. H., Lee, T. M., Li, Y. H. \& Chang, S. F. (2000a). Two new gene cassettes, $d f r 17$ (for trimethoprim resistance) and aadA4 (for spectinomycin/streptomycin resistance), inserted in an Escherichia coli class 1 integron. J Antimicrob Chemother 46, 87-89.

Chang, C. Y., Chang, L. L., Chang, Y. H., Lee, T. M. \& Chang, S. F. (2000b). Characterisation of drug resistance gene cassettes associated with class 1 integrons in clinical isolates of Escherichia coli from Taiwan, ROC. J Med Microbiol 49, 1097-1102.

Collis, C. M. \& Hall, R. M. (1995). Expression of antibiotic resistance genes in the integrated cassettes of integrons. Antimicrob Agents Chemother 39, 155-162.

Dalsgaard, A., Forslund, A., Petersen, A. \& 7 other authors (2000). Class 1 integron-borne, multiple-antibiotic resistance encoded by a 150 kilobase conjugative plasmid in epidemic Vibrio cholerae O1 strains isolated in Guinea-Bissau. J Clin Microbiol 38, 3774-3779. 
Daly, M. \& Fanning, S. (2000). Characterization and chromosomal mapping of antimicrobial resistance genes in Salmonella enterica serotype typhimurium. Appl Environ Microbiol 66, 4842-4848.

Daly, M., Buckley, J., Power, E., O'Hare, C., Cormican, M., Cryan, B. Wall, P. G. \& Fanning, S. (2000). Molecular characterization of Irish Salmonella enterica serotype typhimurium: detection of class I integrons and assessment of genetic relationships by DNA amplification fingerprinting. Appl Environ Microbiol 66, 614-619.

Echeita, M. A., Herrera, S. \& Usera, M. A. (2001). Atypical, $f l j B$-negative Salmonella enterica subsp. enterica strain of serovar 4,5,12:i:- appears to be a monophasic variant of serovar Typhimurium. J Clin Microbiol 39, 2981-2983.

Guard-Petter, J. (2001). The chicken, the egg and Salmonella enteritidis. Environ Microbiol 3, 421-430.

Guerra, B., Soto, S. M., Arguelles, J. M. \& Mendoza, M. C. (2001). Multidrug resistance is mediated by large plasmids carrying a class 1 integron in the emergent Salmonella enterica serotype [4,5,12:i:-]. Antimicrob Agents Chemother 45, 1305-1308.

Hall, R. M. \& Collis, C. M. (1995). Mobile gene cassettes and integrons: capture and spread of genes by site-specific recombination. Mol Microbiol 15, 593-600.

Hall, R. M. \& Stokes, H. W. (1993). Integrons: novel DNA elements which capture genes by site-specific recombination. Genetica 90, 115-132.

Hall, R. M., Brown, H. J., Brookes, D. E. \& Stokes, H. W. (1994). Integrons found in different locations have identical $5^{\prime}$ ends but variable $3^{\prime}$ ends. J Bacteriol 176, 6286-6294.

Heikkila, E., Skurnik, M., Sundstrom, L. \& Huovinen, P. (1993). A novel dihydrofolate reductase cassette inserted in an integron borne on a Tn21-like element. Antimicrob Agents Chemother 37, 1297-1304.

Heim, U., Tietze, E., Weschke, W., Tschape, H. \& Wobus, U. (1989). Nucleotide sequence of a plasmid born streptothricin-acetyl-transferase gene (sat-1). Nucleic Acids Res 17, 7103.

Huovinen, P. \& Jacoby, G. A. (1991). Sequence of the PSE-1 betalactamase gene. Antimicrob Agents Chemother 35, 2428-2430.

Kapperud, G., Lassen, J., Dommarsnes, K., Kristiansen, B. E., Caugant, D. A., Ask, E. \& Jahkola, M. (1989). Comparison of epidemiological marker methods for identification of Salmonella typhimurium isolates from an outbreak caused by contaminated chocolate. J Clin Microbiol 27, 2019-2024.

Kingsley, R. A. \& Baumler, A. J. (2000). Host adaptation and the emergence of infectious disease: the Salmonella paradigm. Mol Microbiol 36, 1006-1014.

Lévesque, C., Brassard, S., Lapointe, J. \& Roy, P. H. (1994). Diversity and relative strength of tandem promoters for the antibiotic-resistance genes of several integrons. Gene 142, 49-54.

Lévesque, C., Piche, L., Larose, C. \& Roy, P. H. (1995). PCR mapping of integrons reveals several novel combinations of resistance genes. Antimicrob Agents Chemother 39, 185-191.

Mazel, D. \& Davies, J. (1999). Antibiotic resistance in microbes. Cell Mol Life Sci 56, 742-754.
Mazel, D., Dychinco, B., Webb, V. A. \& Davies, J. (2000). Antibiotic resistance in the ECOR collection: integrons and identification of a novel aad gene. Antimicrob Agents Chemother 44, 1568-1574.

Nastasi, A., Mammina, C. \& Cannova, L. (2000). Antimicrobial resistance in Salmonella enteritidis, southern Italy, 1990-1998. Emerg Infect Dis 6, 401-403.

Nield, B. S., Holmes, A. J., Gillings, M. R., Recchia, G. D., Mabbutt, B. C., Nevalainen, K. M. \& Stokes, H. W. (2001). Recovery of new integron classes from environmental DNA. FEMS Microbiol Lett 195, 59-65.

Partridge, S. R., Brown, H. J., Stokes, H. W. \& Hall, R. M. (2001). Transposons Tn1696 and Tn21 and their integrons In4 and In2 have independent origins. Antimicrob Agents Chemother 45, 1263-1270.

Radstrom, P., Skold, O., Swedberg, G., Flensburg, J., Roy, P. H. \& Sundstrom, L. (1994). Transposon Tn5090 of plasmid R751, which carries an integron, is related to $T n 7, M u$, and the retroelements. J Bacteriol 176, 3257-3268.

Rowe-Magnus, D. A., Guerout, A. M., Ploncard, P., Dychinco, B., Davies, J. \& Mazel, D. (2001). The evolutionary history of chromosomal super-integrons provides an ancestry for multiresistant integrons. Proc Natl Acad Sci U S A 98, 652-657.

Sandvang, D. (1999). Novel streptomycin and spectinomycin resistance gene as a gene cassette within a class 1 integron isolated from Escherichia coli. Antimicrob Agents Chemother 43, 3036-3038.

Sandvang, D., Aarestrup, F. M. \& Jensen, L. B. (1997). Characterisation of integrons and antibiotic resistance genes in Danish multiresistant Salmonella enterica Typhimurium DT104. FEMS Microbiol Lett 157, 177-181.

Shaw, K. J., Rather, P. N., Hare, R. S. \& Miller, G. H. (1993). Molecular genetics of aminoglycoside resistance genes and familial relationships of the aminoglycoside-modifying enzymes. Microbiol Rev 57, 138-163.

Stokes, H. W. \& Hall, R. M. (1989). A novel family of potentially mobile DNA elements encoding site-specific gene-integration functions: integrons. Mol Microbiol 3, 1669-1683.

Sunde, M. \& Sørum, H. (1999). Characterization of integrons in Escherichia coli of the normal intestinal flora of swine. Microb Drug Resist 5, 279-287.

Tosini, F., Visca, P., Luzzi, I., Dionisi, A. M., Pezzella, C., Petrucca, A. \& Carattoli, A. (1998). Class 1 integron-borne multiple-antibiotic resistance carried by IncFI and IncL/M plasmids in Salmonella enterica serotype typhimurium. Antimicrob Agents Chemother 42, 3053-3058.

Tschape, H., Tietze, E., Prager, R., Voigt, W., Wolter, E. \& Seltmann, G. (1984). Plasmid-borne streptothricin resistance in gram-negative bacteria. Plasmid 12, 189-196.

White, P. A., Mclver, C. J., Deng, Y. \& Rawlinson, W. D. (2000). Characterisation of two new gene cassettes, aadA5 and dfrA17. FEMS Microbiol Lett 182, 265-269.

Zhao, S., White, D. G., Ge, B., Ayers, S., Friedman, S., English, L., Wagner, D., Gaines, S. \& Meng, J. (2001). Identification and characterization of integron-mediated antibiotic resistance among Shiga toxinproducing Escherichia coli isolates. Appl Environ Microbiol 67, $1558-1564$. 